\section{Estandarización de la fuerza de prensión manual en adultos chilenos sanos mayores de 20 años}

\author{
CAROLINA ROMERO-DAPUETO ${ }^{1, \mathrm{a}}$, JESSICA MAHN ${ }^{2, \mathrm{~b}}$, \\ GABRIEL CAVADA $^{3, \mathrm{c}}$, RODRIGO DAZA ${ }^{1, \mathrm{a}}$, \\ VÍCTOR ULLOA ${ }^{1, a}$, MARCELA ANTÚNEZ
}

\section{Hand grip strength values in normal Chilean subjects}

Background: A low hand grip strength is a prognostic factor both in healthy people and hospitalized subjects. Local normal hand grip strength values are needed to define cutoff points of abnormality. Aim: To measure handgrip strength in Chilean people aged 20 to 70 years and propose normal values for healthy people in this age range. Material and Methods: Handgrip strength was measured using a JAMAR hydraulic dynamometer in 436 males and 465 females aged 20 to 70 years and who were free of disease. They were recruited from waiting rooms in several public and private hospitals and outpatient clinics, under self-evaluation of inclusion criteria. Results: The variability of the handgrip strength in women was smaller in than men. Tables containing handgrip strength values by age and sex and the ranges between three standard deviations were prepared. Conclusions: This study contributes with normal handgrip strength values in Chile to be used for the diagnosis and management of various conditions, such sarcopenia, obesity, oncological patients, Intensive Care Unit acquired weakness (ICU-aw) and weaning of mechanical ventilation.

(Rev Med Chile 2019; 147: 741-750)

Key words: Hand Strength; Reference Values; Rehabilitation.

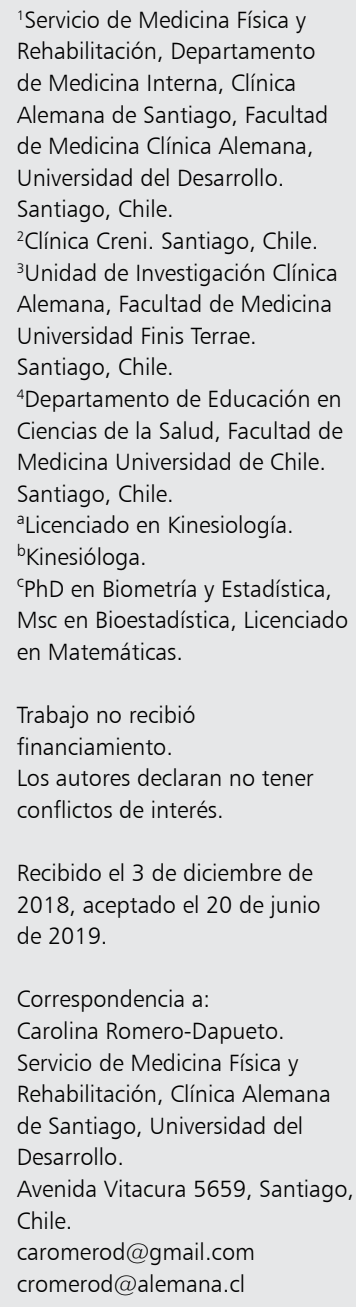

Recibido el 3 de diciembre de 2018, aceptado el 20 de junio de 2019.

Correspondencia a: Carolina Romero-Dapueto. Servicio de Medicina Física y Rehabilitación, Clínica Alemana de Santiago, Universidad del Desarrollo. Avenida Vitacura 5659, Santiago, Chile. caromerod@gmail.com cromerod@alemana.cl

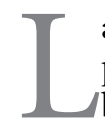

a medición de la fuerza muscular es un parámetro de utilidad para objetivar rehabilitación y medir su comportamiento frente a una determinada condición clínica. Para este fin, la medición objetiva de la fuerza de prensión permite la evaluación de la funcionalidad de la mano, estimando valores que se pueden utilizar para determinar el resultado de un manejo médico, ortopédico o quirúrgico. Además, se puede estimar cuándo un sujeto está en condiciones de volver a sus actividades habituales, o dando un factor pronóstico en cuanto a mortalidad.

La medición de la fuerza de prensión se realiza comúnmente mediante la dinamometría, la que puede ser con un dinamómetro hidráulico o digital. La dinamometría hidráulica es la forma más común de evaluación, la cual se estima con el dinamómetro Jamar (Jamar ${ }^{T M}$ Hidraulic Hand Dynamometer, Preston, Jackson, Missouri. $E E U U)$. Este es un procedimiento fácil de realizar y reproducible. Este instrumento mide la fuerza isométrica y presenta cinco posiciones diferentes para el agarre. Su uso es apropiado, ya que es el propio sujeto el que controla su fuerza, en ausencia de intervenciones externas (ejercidas por el examinador). La American Society of Hand Therapist (ASHT) recomienda la posición II para la medición de la fuerza máxima. Esta posición es 
el criterio recomendado para estudios donde se busca obtener datos normativos ${ }^{1}$.

A nivel nacional existen pocos estudios de medición de la fuerza de prensión. El de Gómez y cols. no es extrapolable a toda la población por ser de demanda laboral de la extremidad superior ${ }^{2}$, el de Mansilla en el 2016 sólo abarca población de adultos mayores entre 60 y 91 años $^{3}$ y el de Gómez Campos abarca población entre 6 y 17,9 años ${ }^{4}$. Actualmente esta evaluación se realiza comparando la fuerza de la mano afectada con la mano contralateral, siempre y cuando esté libre de patologías. Este procedimiento carece en ocasiones de validez, por ejemplo, en el caso de presentarse patologías en ambas extremidades superiores, pacientes amputados de extremidad superior, artefactos de medición en las extremidades (líneas arteriales y vías venosas) o poca confiabilidad respecto a la dominancia en el sujeto evaluado (ejemplo, patologías que presenten alteración cognitiva, como delirium o Alzheimer). Es por esto, que se hace necesario disponer de una tabla de referencia con valores normales. Los resultados de diferentes estudios apoyan de igual forma la poca validez de la comparación con la mano contralateral ${ }^{5,6}$. En más de $95 \%$ de los sujetos sanos la diferencia de fuerza entre ambas manos es igual o menor de $10 \% \%^{5-9}$.

Se ha observado que en hombres como en mujeres, la fuerza de puño comienza a disminuir lentamente con la edad, obteniéndose un máximo de mayor fuerza entre los 30 y los 50 años, para luego disminuir gradualmente. Este comportamiento es similar entre hombres y mujeres y los factores causales de la disminución de la fuerza difieren entre ambos sexos. En los hombres, esta disminución se debe a una gradual pérdida de la capacidad física y a un aumento del sedentarismo, con los subsiguientes cambios en las fibras musculares. En cambio, en las mujeres, las causas son los parámetros antropométricos (altura y peso), el sedentarismo y los cambios hormonales ${ }^{10,11}$. Otro factor que disminuye la fuerza de prensión son las causas infecciosas, por ejemplo la sepsis, llegando a una disminución de $35 \%$ de la fuerza basal, lo que podría ser de gran interés en condiciones actuales patológicas como la debilidad adquirida en $\mathrm{UCI}^{12,13}$.

Por estos motivos, se hace necesaria la existencia de valores normales para la evaluación de un paciente en ciertos contextos clínicos. Es por esto que el objetivo del presente trabajo es determinar los valores normales de fuerza de puño en población sin patologías conocidas de extremidades superiores, permitiéndonos tener una referencia que pueda ser utilizada en la práctica clínica diaria del médico, del kinesiólogo y de otros profesionales de la salud en Chile. Este tipo análisis permitirá la confección de tablas de normalidad, para optimizar las evaluaciones clínicas y determinar objetivamente el grado de recuperación de la fuerza de puño, asociado a factores pronósticos en distintas poblaciones de pacientes adultos.

\section{Material y Método}

Se estudiaron 901 sujetos de ambos sexos, sin patologías conocidas de las extremidades superiores, reclutados entre junio de 2006 y septiembre de 2018. Para calcular los niveles de fuerza de puño estandarizados ( $z$ score de fuerza de puño) se utilizó una regresión lineal con la cual se predijo los residuos estandarizados los que constituyen el $z$ score, para estimar la regresión se utilizó la regla de 10 sujetos por variable, dado que son siete rangos etarios y dos sexos se consideró tener como mínimo 200 sujetos, pero sin embargo, para una mayor precisión en las estimaciones, se evaluaron 436 hombres y 465 mujeres. Los criterios de inclusión fueron sujetos entre 20 y 70 años de edad que vivan en Chile, con ausencia de dolor reciente en las manos, brazos y columna cervical, con capacidad de realizar sus actividades habituales sin alguna restricción por algún problema de salud. Los criterios de exclusión fueron patologías que afecten la fuerza de sus extremidades superiores como por ejemplo, Síndrome del Túnel Carpiano, salvo procesos involutivos secundarios propios de la edad, sujetos que presenten alteraciones en la sensibilidad de la mano, lesiones neurológicas periféricas, que presenten enfermedades sistémicas metabólicas, como artritis reumatoide, diabetes mellitus, presencia de dolor inespecífico en la extremidad superior, haber transcurrido menos 6 meses desde el alta de una hospitalización por cualquier otro proceso, realización habitual de trabajos de alta demanda de extremidad superior $\mathrm{y}$ deportistas profesionales.

Los sujetos del estudio se muestrearon en forma no probabilística de entre sujetos que estaban en salas de espera para atención médica en Clí- 
nica Alemana y Clínica Santa María de Santiago, Hospital Instituto Traumatológico de Santiago, Centro Médico MEGASALUD de San Bernardo, alumnos y personal de la Facultad de Medicina de la Universidad de Chile y de la Universidad del Desarrollo. A los individuos se les invitaba a medir la fuerza de mano y se verificaba que cumplieran los criterios de inclusión, mediante el llenado de una ficha de autorreporte. La muestra se completó considerando igual frecuencia por rango etario quinquenal desde los 20 a los 65 años. Los sujetos fueron registrados de forma anónima. El protocolo fue aprobado por el Departamento Científico Docente del Instituto Traumatológico Dr. Teodoro Gebauer.

Para la medición de la fuerza de prensión manual se utilizó un dinamómetro hidráulico marca JAMAR ${ }^{\circledR}\left(\right.$ Jamar $^{T M}$ Hidraulic Hand Dynamometer, Preston, Jackson, Missouri. EEUU) en posición II. Esta evaluación se lleva a cabo con el sujeto sentado en una silla con respaldo, hombros aducidos y sin rotación, codo flectado en $90^{\circ}$, antebrazo en posición neutra y muñeca en posición neutra (En extensión entre $0-30^{\circ}$ y con una desviación ulnar de $0^{\circ}-15^{\circ}$ ), con ambos pies apoyados en el suelo y con la espalda apoyada en el respaldo. El brazo evaluado no se apoya en superficie alguna y el dinamómetro se utiliza en posición vertical. El participante realizaba una fuerza de prensión máxima durante 3 segundos, con reposo de $1 \mathrm{~min}$ entre cada repetición, realizando dos intentos.

\section{Análisis estadístico}

Las variables categóricas fueron descritas mediante frecuencias y proporciones. Las variables numéricas fueron descritas mediante promedio y desviación estándar. La homogeneidad de la muestra (independencia entre sexo y rango etario) fue docimada a través del test de independencia de $\chi^{2}$. Mediante un análisis de regresión lineal múltiple se asoció la máxima fuerza, de entre la mano izquierda y derecha, con las variables explicativas: sexo y edad, luego se estimó el residuo estandarizado de cada unidad de observación ( $z$ score), la normalidad de dichos residuos y de las fuerzas medidas se docimó mediante el test de Shapiro-Wilk. Los $z$ score fueron tabulados en función de la fuerza, edad y sexo, destacándose los valores de fuerza comprendidos entre - 3 y 3 desviaciones estándar, además de los valores medios $(z$ score $=0)$ y los valores de fuerza comprendidos entre - 1 y 1 desviaciones estándar. Todos los intervalos de confianza fueron de nivel $95 \%$ y se utilizó un nivel de significación de 5\%. Los datos fueron procesados en STATA versión 14.0.

\section{Resultados}

Se reclutaron 901 sujetos de los cuales 465 $(51,6 \%)$ fueron de sexo femenino. La Tabla 1 muestra la distribución etaria y por sexo de los sujetos analizados.

Dado que la prueba de asociación entre sexo y rango etario no fue estadísticamente significativa, se obtuvo una muestra comparable por sexo y edad.

La Tabla 2 muestra las estadísticas descriptivas de la fuerza, kilógramos, por rango etario y sexo.

La Tabla 2, muestra que la fuerza media ejercida por hombres es significativamente mayor en hombres que en mujeres $(p=0,0000)$ en todas las edades, dado que se observan coeficientes de variabilidad menores al 25\% las fuerzas medidas por rango etario y al interior de cada sexo son homogéneas, no hay evidencia para descartar que las fuerzas, por rango etario y sexo, tengan distribución normal o gaussiana, excepto en el último rango etario de las mujeres debido al valor extremo de 52 kilógramos en dicho rango.

La Tabla 3, muestra los coeficientes de la regresión lineal múltiple que explica la fuerza por edad y sexo.

La Tabla 3 muestra que las mujeres en promedio ejercen una fuerza de 17 kilos menor que los hombres, ajustando por edad, $y$, que por cada año de edad se evidencia una pérdida de fuerza de 0,2 kilos ajustando por sexo. Esta regresión explica la fuerza con un coeficiente de determinación $\mathrm{R}^{2}=64,7 \%$, y el coeficiente de correlación entre las fuerzas observadas y predichas es de $80,6 \%$. A partir de este modelo se obtuvieron los residuos estandarizados con los cuales se construyó la tabla 4 que contiene los $z$-scores de fuerza por sexo y edad. No se puede descartar la normalidad de los residuos de la regresión propuesta $(\mathrm{p}=0,9698)$.

Las Tablas 4 y 5 muestran los valores normativos en ambos sexos, destacando el valor medio, y desde una a tres desviaciones estándar, para todas las edades, en ambos sexos. 
Tabla 1. Distribución etaria de los sujetos reclutados por sexo

\begin{tabular}{|c|c|c|c|c|c|c|}
\hline \multirow{2}{*}{$\begin{array}{l}\text { Rango } \\
\text { etario }\end{array}$} & \multicolumn{2}{|c|}{ Masculino } & \multicolumn{2}{|c|}{ Femenino } & \multicolumn{2}{|c|}{ Total } \\
\hline & $\mathbf{n}$ & $\%$ & $\mathbf{n}$ & $\%$ & $\mathbf{n}$ & $\%$ \\
\hline $20-24$ & 44 & 10,1 & 44 & 9,5 & 88 & 9,8 \\
\hline $25-29$ & 41 & 9,4 & 47 & 10,1 & 88 & 9,8 \\
\hline $30-34$ & 48 & 11,0 & 47 & 10,1 & 95 & 10,5 \\
\hline $35-39$ & 47 & 10,8 & 51 & 11,0 & 98 & 10,9 \\
\hline $40-44$ & 42 & 9,6 & 55 & 11,8 & 97 & 10,8 \\
\hline $45-49$ & 44 & 10,1 & 47 & 10,1 & 91 & 10,1 \\
\hline $50-54$ & 41 & 9,4 & 42 & 9,0 & 83 & 9,2 \\
\hline $55-59$ & 44 & 10,1 & 46 & 9,9 & 90 & 10,0 \\
\hline $60-64$ & 43 & 9,9 & 43 & 9,2 & 86 & 9,5 \\
\hline $65-70$ & 42 & 9,6 & 43 & 9,2 & 85 & 9,4 \\
\hline Total & 436 & 100,0 & 465 & 100,0 & 901 & 100,0 \\
\hline
\end{tabular}

p-value $=0,997$ para la asociación de sexo y rango etario.

Tabla 2. Fuerza en kilógramos por rango etario y sexo (número de sujetos, mínimo, cuartiles, máximo, promedio, desviación estándar, coeficiente de variabilidad y p-value para la normalidad de los datos)

\begin{tabular}{|ccccccccccc|}
\hline Edad & $\mathbf{n}$ & $\mathbf{m i n}$ & $\mathbf{p 2 5}$ & $\mathbf{p 5 0}$ & $\mathbf{p 7 5}$ & $\mathbf{m a x}$ & Promedio & de & cv (\%) & p-value \\
Hombres & & & & & & & & & & \\
$20-24$ & 44 & 28 & 47,5 & 51 & 59 & 70 & 51,6 & 9,6 & 18,6 & 0,2861 \\
$25-29$ & 41 & 32 & 44 & 50 & 53 & 69 & 49,2 & 7,0 & 14,3 & 0,9127 \\
$30-34$ & 48 & 32 & 46 & 52 & 55,5 & 68 & 50,6 & 7,6 & 15,0 & 0,4828 \\
$35-39$ & 47 & 34 & 46 & 49 & 54 & 66 & 49,3 & 6,6 & 13,4 & 0,9999 \\
$40-44$ & 42 & 36 & 46 & 49,5 & 56 & 68 & 50,9 & 7,5 & 14,7 & 0,6402 \\
$45-49$ & 44 & 30 & 42 & 48 & 52,5 & 61 & 47,8 & 7,3 & 15,4 & 0,9419 \\
$50-54$ & 41 & 29 & 41 & 45 & 51 & 58 & 45,5 & 6,7 & 14,7 & 0,1533 \\
$55-59$ & 44 & 33 & 42 & 46,5 & 52 & 60 & 46,6 & 6,5 & 13,9 & 0,7234 \\
$60-64$ & 43 & 36 & 43 & 47 & 50 & 60 & 46,3 & 5,1 & 11,1 & 0,1596 \\
$65-70$ & 42 & 24 & 35 & 41 & 44 & 52 & 39,3 & 7,5 & 19,0 & 0,1025 \\
Total & 436 & 24 & 43 & 48 & 53 & 70 & 47,8 & 7,9 & 16,6 \\
Mujeres & & & & & & & & & \\
$20-24$ & 44 & 18 & 28 & 30 & 34,5 & 48 & 31,1 & 5,2 & 16,7 & 0,3313 \\
$25-29$ & 47 & 20 & 28 & 33 & 36 & 47 & 32,5 & 5,5 & 17,0 & 0,9477 \\
$30-34$ & 47 & 24 & 28 & 32 & 36 & 45 & 32,4 & 5,0 & 15,4 & 0,6854 \\
$35-39$ & 51 & 22 & 29 & 34 & 39 & 48 & 34,1 & 6,3 & 18,5 & 0,7510 \\
$40-44$ & 55 & 19 & 27 & 30 & 35 & 44 & 31,0 & 5,5 & 17,6 & 0,7723 \\
$45-49$ & 47 & 21 & 28 & 32 & 36 & 41 & 31,6 & 5,3 & 16,8 & 0,6792 \\
$50-54$ & 42 & 19 & 28 & 30 & 33 & 41 & 30,8 & 4,5 & 14,6 & 0,9524 \\
$55-59$ & 46 & 16 & 23 & 28 & 31 & 41 & 27,8 & 5,6 & 20,3 \\
$60-64$ & 43 & 20 & 26 & 29 & 31 & 36 & 28,6 & 3,4 & 11,9 & 0,9273 \\
$65-70$ & 43 & 16 & 22 & 27 & 32 & 52 & 27,0 & 6,6 & 24,3 \\
Total & 465 & 16 & 27 & 30 & 34 & 52 & 30,8 & 5,7 & 18,6 & 0,0077 \\
\hline
\end{tabular}

Tabla 3. Coeficientes de regresión de edad y sexo

\begin{tabular}{|ccccc|}
\hline Fuerza & Coeficiente & p-value & \multicolumn{2}{c|}{ Intervalo de Confianza } \\
Femenino & $-17,0$ & 0,0000 & $-17,9$ & $-16,2$ \\
Edad & $-0,2$ & 0,0000 & $-0,2$ & $-0,1$ \\
\hline
\end{tabular}




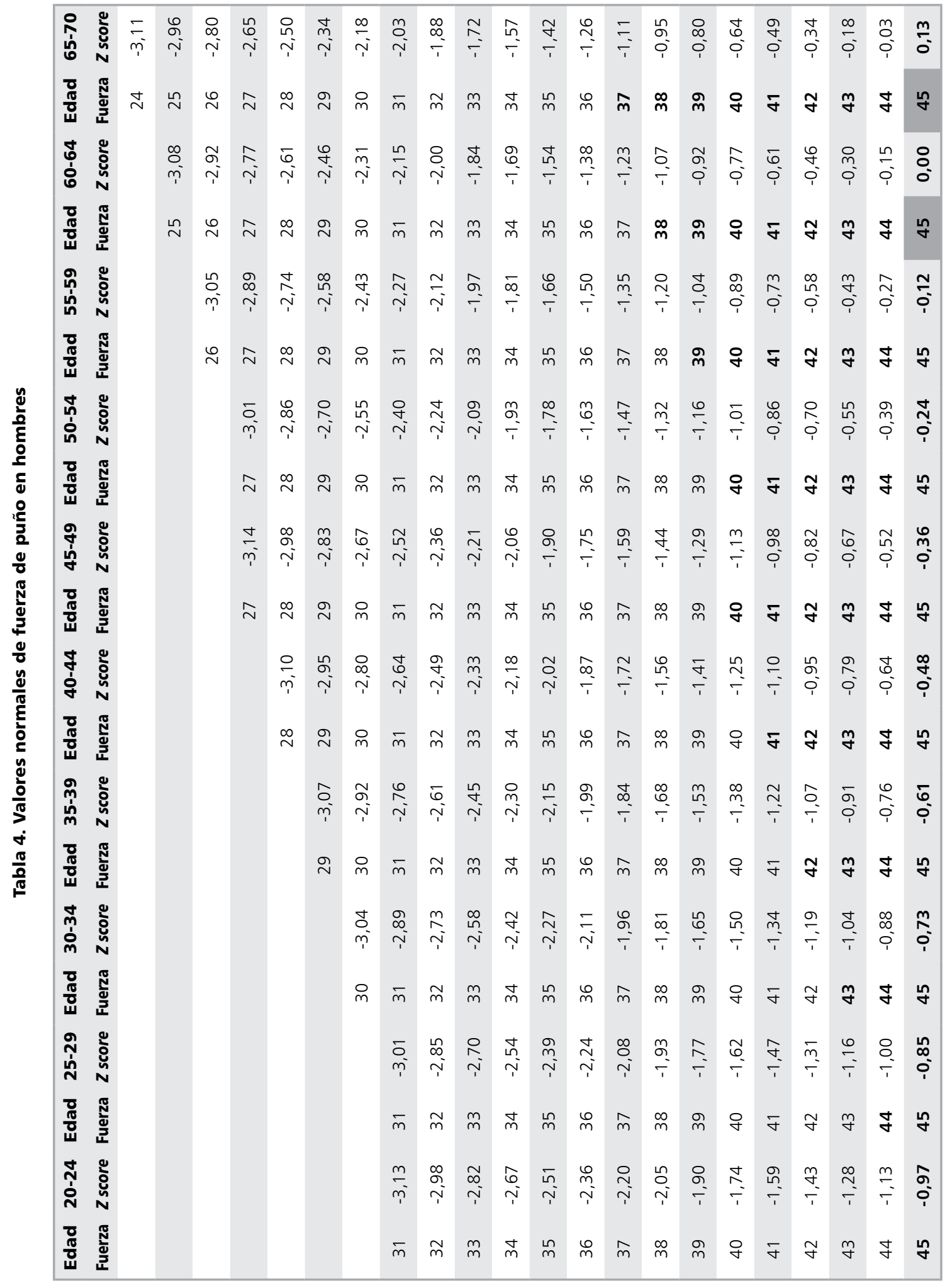




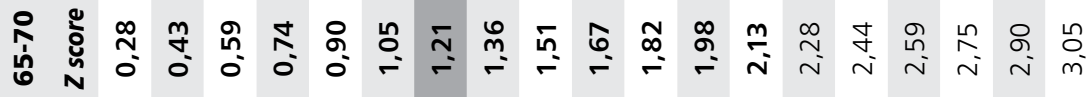

ரृ

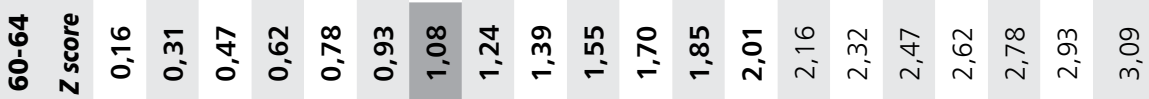

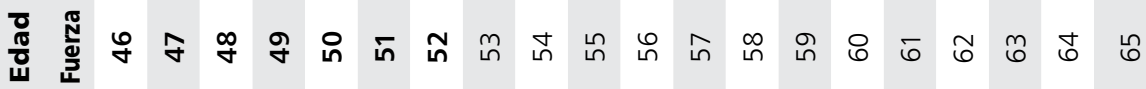

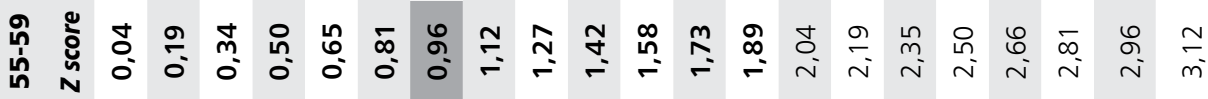

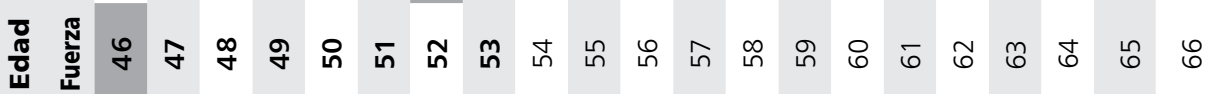

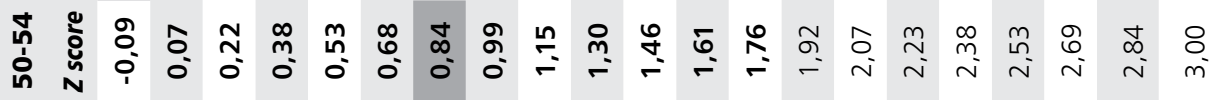

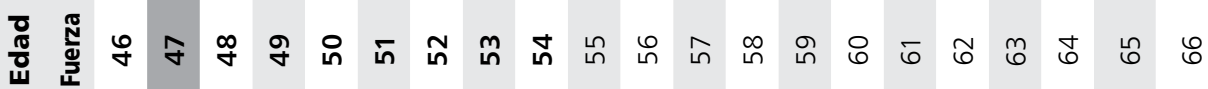

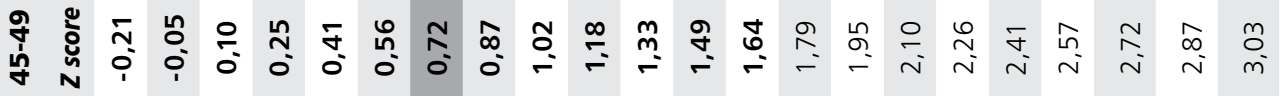

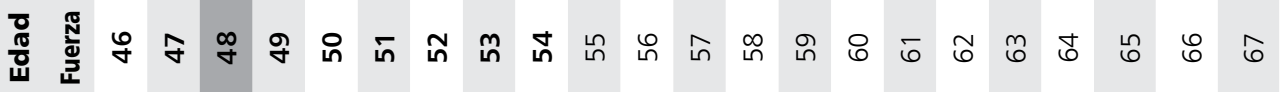

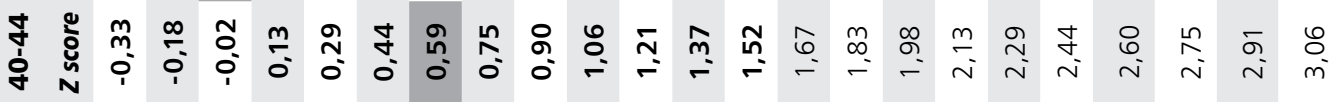

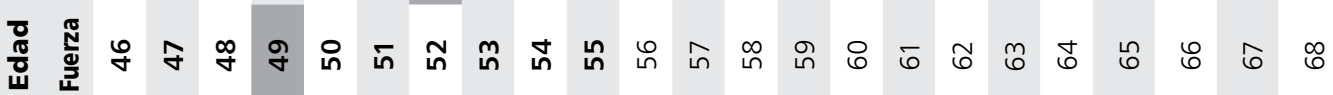

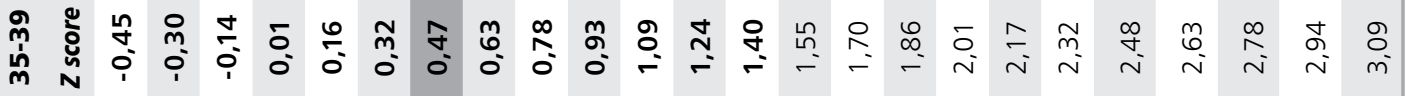

焉

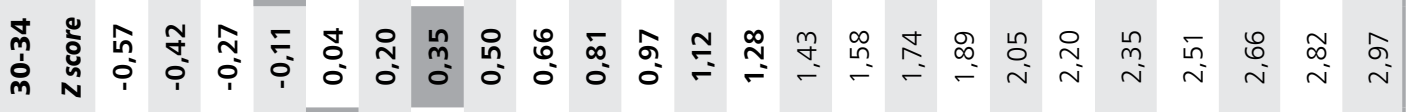

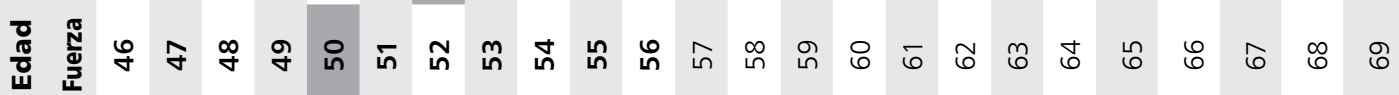

衣总

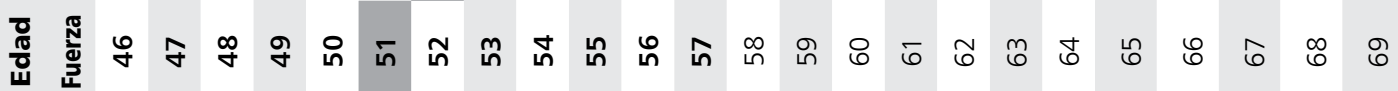

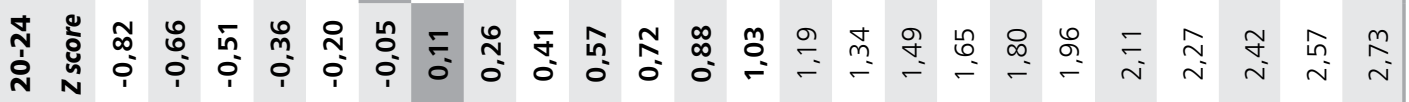

宽 总 


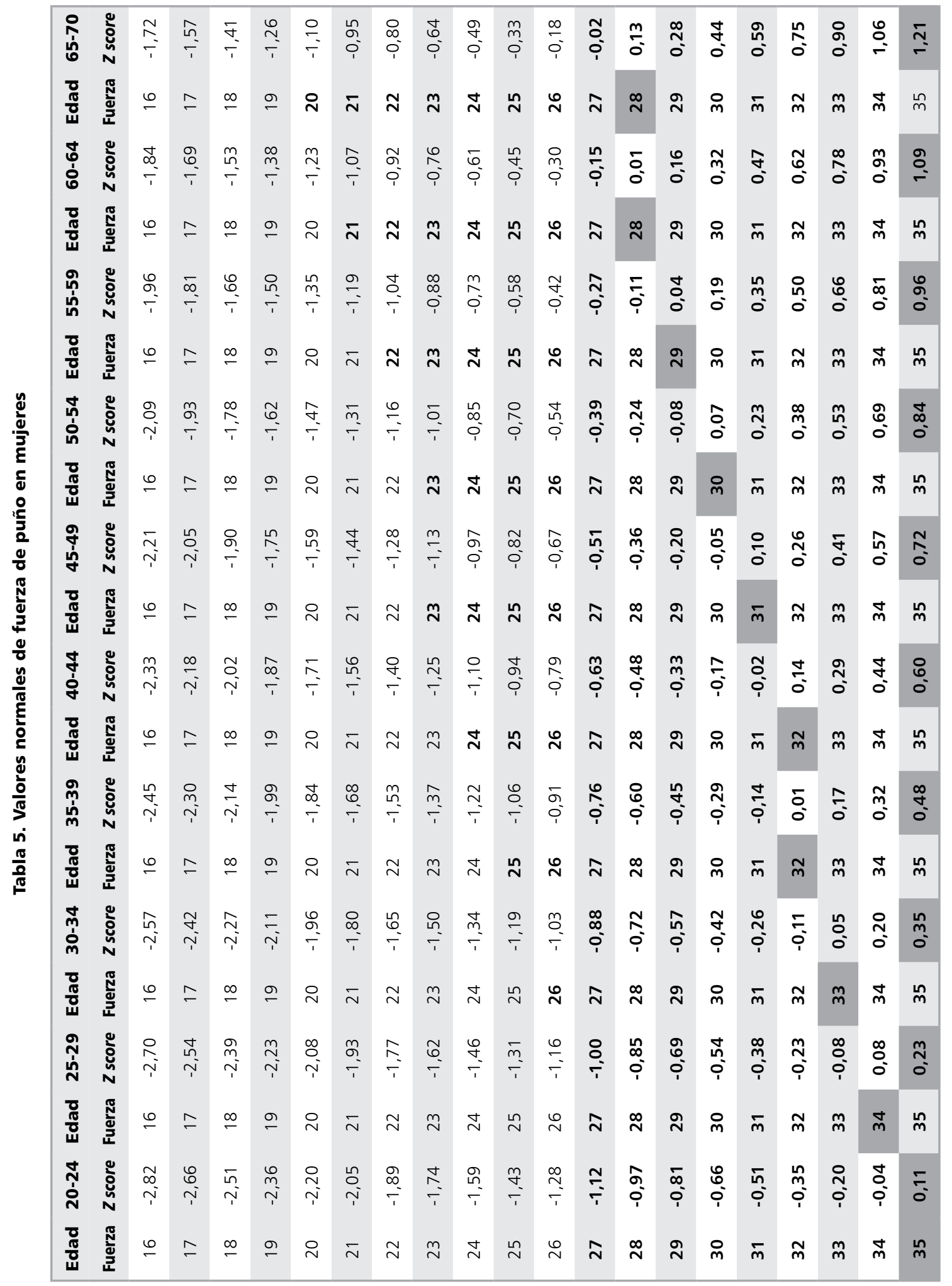




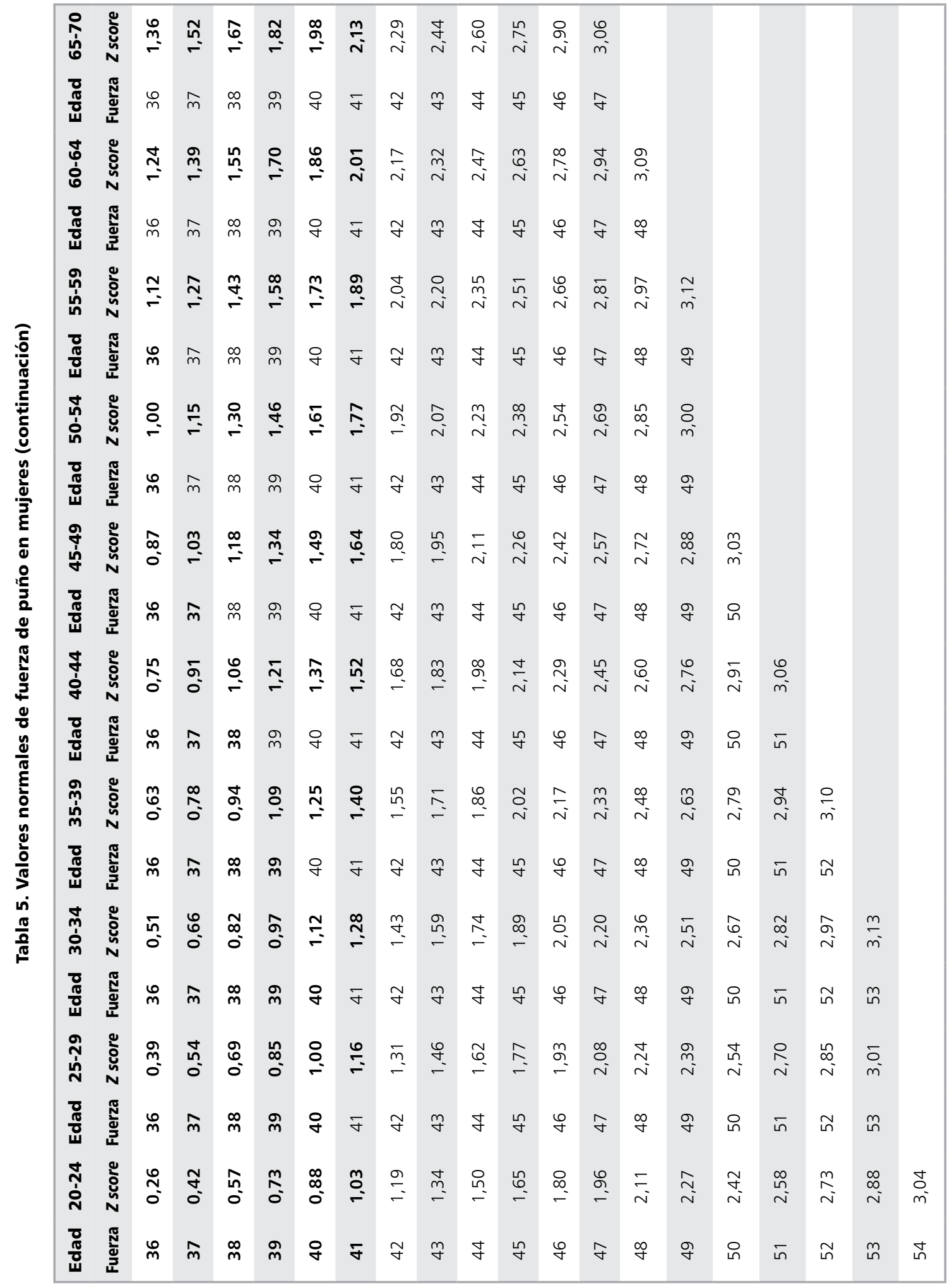




\section{Discusión}

La medición de la fuerza muscular manual a través de la dinamometría ha sido de gran interés últimamente, ya que sólo medir esta variable funcional se asocia al desenlace clínico en diversas patologías. Hay una asociación entre la fuerza muscular y calidad de vida en distintas poblaciones, donde los adultos con deterioro en la fuerza muscular tienen limitación en sus actividades de la vida diaria (AVD) y como consecuencia restricción en su participación, como la actividad física recreativa, generando una mayor asistencia familiar y social. Además, estas personas son frágiles y más vulnerables a accidentes como caídas u otros eventos adversos por lo tanto la recuperación de alguna patología aguda, lesión o cirugía puede estar comprometida o enlentecida. Este estudio presenta datos normativos de valores de referencia para la fuerza de prensión manual. Estos resultados deben ser beneficiosos para diversas condiciones clínicas y ergonómicas siendo capaz de comparar valores en individuos con o sin disminución de la fuerza en relación a la población general en Chile. Determinar estos valores de referencias ayudará a los profesionales de la salud para documentar la progresión del proceso de rehabilitación y además de proveer retroalimentación con los valores normales según sexo y edad. En relación al género, se observa una mayor fuerza de prensión manual en hombres con respecto a las mujeres en todos los rangos etarios similar a lo documentado por Mancilla y $\mathrm{col}^{3,13}$. En cuanto al descenso de fuerza que se observa con la edad es homogéneo tanto en hombres como mujeres.

Esta medición se ha correlacionado al resultado clínico en una amplia variedad de patologías. Un estudio encontró que niveles bajos de fuerza de puño fueron un factor independientemente asociado a mayores días en ventilación mecánica, mayores días de estadía hospitalaria, mayor mortalidad intrahospitalaria y peor resultado en la movilidad funcional en paciente ingresados a UCI y además clasificó a esta población de pacientes según el criterio clínico de debilidad adquirida en UCI (fuerza prensión manual mujeres $<7 \mathrm{~kg}$ y hombre $<11 \mathrm{~kg})^{14}$. Otros estudios han confirmado el vínculo entre fuerza muscular, pronóstico y complicaciones posterior a cirugías tanto médicas como quirúrgicas ${ }^{16}$, siendo un ejemplo la cirugía bariátrica la cual a través de esta medición per- mite identificar a pacientes que necesiten mayor atención sobre la optimización de la composición corporal previo a la cirugía ${ }^{20}$. En pacientes oncológicos, niveles bajos de fuerza de puño fueron un factor independiente asociado con la sobrevida y una peor calidad de vida ${ }^{15}$. Además, la fuerza muscular también es un predictor independiente de todas las causas de mortalidad en una población sana, siendo mayor en el sexo femenino ${ }^{16-18}$. En pacientes geriátricos con criterio clínico de sarcopenia (fuerza prensión manual $<30 \mathrm{~kg}$ en hombre $\mathrm{y}<20 \mathrm{~kg}$ en mujeres) la fuerza de prensión manual se correlaciona negativamente con mortalidad independiente de la edad, condición clínica y variables funcionales ${ }^{19}$.

Futuras líneas de investigación debiesen enfocarse a la evaluación de este dominio del rendimiento físico, pudiendo servir como un parámetro objetivo o incluirse en evaluaciones desde edades tempranas para utilizarla como método preventivo gracias a su fácil aplicabilidad y reproducibilidad.

Una limitación del presente estudio es que en las tablas de normalidad sólo se presenta un valor único de fuerza máxima y hasta tres desviaciones estándar para la comparación de las fuerzas. Esto porque en gran parte de los estudios la diferencia entre la fuerza de ambas manos es menor al 10\% y al tener un valor único de fuerza se asume que ese valor es el de la mano dominante. En nuestra estandarización, la diferencia de la fuerza entre ambas manos fue de 4,2\%. Esto tiene sentido en un proceso de rehabilitación, ya que al medir la fuerza de prensión, esta mejora en ambas manos indistintamente de la dominancia del sujeto. Con respecto a la fuerza y la dominancia, en la mayoría de los estudios la mano derecha presenta mejor rendimiento que la mano izquierda, y así lo demuestra la evidencia disponible, donde la mano derecha es más fuerte que la izquierda ${ }^{22}$.

Basados en el actual conocimiento científico disponible, el alto riesgo de mortalidad asociado a bajos niveles de fuerza de prensión manual, es similar a otros factores de riesgo bien establecidos para muerte prematura como lo son la obesidad, hipertensión arterial y el consumo de tabaco. Por esta razón, diversas sociedades científicas recomiendan que cada individuo realice un entrenamiento de fuerza muscular como mínimo 2 a 3 veces a la semana en días no consecutivos ${ }^{21}$. De esta forma, podemos concluir que los valores 
de referencia normativos aquí proporcionados pueden servir como una guía para interpretar la medición de fuerza de prensión manual obtenidas en adultos sanos de la población chilena.

\section{Referencias}

1. Boadella JM, Kuijer PP, Sluiter JK, Frings-Dresen MH. Frings-Dresen.2005. Effect of self- selected handgrip position on maximal handgrip strength. Arch Phys Med Rehabil 86 (2): 328-31.

2. Gómez S, Navarrete E, Sepúlveda I, Lobos E. Datos normativos de la fuerza de agarre en sujetos en edad laboral en el Área Metropolitana. Revista Chilena de Rehabilitación de la Mano 1998; 1: 7-1.

3. Mancilla SE, Ramos FS, Morales BP. Fuerza de prensión manual según edad, género y condición funcional en adultos mayores Chilenos entre 60 y 91 años. Rev Med Chile 2016; 144: 598-603.

4. Gómez-Campos R, Andruske CL, Arruda M, Sulla-Torres J, Pacheco-Carrillo J, Urra-Albornoz C, et al. Normative data for handgrip strength in children and adolescents in the Maule Region, Chile: Evaluation based on chronological and biological age. PLoS One 2018; 13 (8).

5. Bechtol CO. Grip test: Use of dynamometer with adjustable handle spacings. J Bone Joint Surg 1954; 36A: 820-4.

6. Josty IC, Tyler MP, Shewell PC, Roberts AH. Grip and pinch strength variations in differents types of workers. J Hand Surg Br 1997; 22 (2): 266-9.

7. Mathiowetz V, Kashman N, Volland G, Weber K, Dowe M, Rogers S. Grip and pinch strength: Normative data for adults. Arch Phys Med Rehabil 1985; 66: 69-74.

8. Schmidt RT, Toews JV. Grip strength as measured by the Jamar dynamometer. Arch Phys Med Rehabil 1970; 51: 321-7.

9. Torres Coscoyuela M, González del Pino J, Yáñez Calvo J, Bartolomé del Valle E. Estudio dinamométrico de la mano y el pulgar. Rev Chil Ortop y Traum 1999; 43: 321-6.

10. Cauley JA, Petrini AM, LaPorte RE, Sandler RB, Bayles $\mathrm{CM}$, Robertson RJ, et al. The decline of grip strength in the menopause: relationship to physical activity, estrogen use and antropometric factors. J Chronic Dis 1987; 40 (2): 115-20.
11. Crosby C, Wehbe M. Hand strength: normative values. J Hand Surg 1994; 19A: 650-70.

12. Martin S, Neale G, Elia M. Factors affecting maximal momentary grip strength. Hum Nutr Clin Nutr 1985; 39 (2): 137-47.

13. Wang YC, Bohannon RW, Li X, Sindhu B, Kapellusch J. Hand Grip Strength: Normative Reference Values and Equations for 18- to 85-Year-Olds Residing in the United States. J Orthop Sports Phys Ther 2018; 48 (9): 685-93.

14. Ali NA, O'Brien JM Jr, Hoffmann SP, Phillips G, Garland A, Finley JC, et al. Acquired weakness, handgrip strength, and mortality in critically ill patients. Am J Respir Crit Care Med 2008; 178 (3): 261-8.

15. Kilgour RD, Vigano A, Trutschnigg B, Lucar E, Borod $\mathrm{M}$, Morais JA. Handgrip strength predicts survival and is associated with markers of clinical and functional outcomes in advanced cancer patients. Support Care Cancer 2013; (12): 3261-70.

16. Volaklis KA, Halle M, Meisinger C. Muscular strength as a strong predictor of mortality: A narrative review. Eur J Intern Med 2015; (5): 303-10.

17. García-Hermoso A, Cavero-Redondo I, Ramírez-Vélez R, Ruiz JR, Ortega FB, Lee DC, et al. Muscular Strength as a Predictor of All-Cause Mortality in an Apparently Healthy Population: A Systematic Review and Meta-Analysis of Data From Approximately 2 Million Men and Women. Arch Phys Med Rehabil 2018; 10: 21002113.e5.

18. Leong DP, Teo KK, Rangarajan S, López-Jaramillo P, Avezum A Jr, Orlandini A, et al. Prognostic value of grip strength: findings from the Prospective Urban Rural Epidemiology (PURE) study. Lancet 2015; 386: 266-73.

19. Landi F, Cruz-Jentoft AJ, Liperoti R, Russo A, Giovannini S, Tosato M, et al. Sarcopenia and mortality risk in frail older persons aged 80 years and older: results from ilSIRENTE study. Age Ageing 2013; 42 (2): 203-9.

20. Otto M. Handgrip strength as a predictor for post bariatric body composition. Obes Surg 2014; (12): 2082-8.

21. ACSM Guidelines for Exercise Testing and Prescription, tenth edition 2018.

22. Bohannon RW, Peolsson A, Massy-Westropp N, Desrosiers J, Bear-Lehman J. Reference values for adult grip strength measured with a Jamar dynamometer: a descriptive meta-analysis. Physiotherapy 2006; 92 (1): 11-5. 